\title{
Long distance electron transfer in cytochrome $c$ oxidase immobilised on electrodes. A surface enhanced resonance Raman spectroscopic study
}

\author{
Jana Hrabakova, ${ }^{a}$ Kenichi Ataka, ${ }^{b}$ Joachim Heberle, ${ }^{b}$ Peter Hildebrandt ${ }^{\star a}$ and \\ Daniel H. Murgida*a
}

Received 6th May 2005, Accepted 9th December 2005

First published as an Advance Article on the web 20th December 2005

DOI: $10.1039 / \mathbf{b 5 1 3 3 7 9 n}$

Cytochrome $c$ oxidase was tethered to a functionalised Ag electrode via a histidine-tag on the C-terminus of subunit I or II and embedded in a phospholipid bilayer. The uniformly oriented membrane-bound proteins were studied by surface enhanced resonance Raman spectroscopy (SERRS) that reveals preservation of the native structures of the heme $a$ and heme $a_{3}$ sites. On the basis of time-dependent SERRS measurements, the rate constant for the heterogeneous electron transfer to heme $a$ was determined to be $0.002 \mathrm{~s}^{-1}$ independent of the enzyme orientation and the overpotential. Taking into account that the electrode-to-heme $a$ distance is larger than 50 $\AA$, these findings suggest an electron hopping mechanism in which the $\mathrm{Cu}_{\mathrm{A}}$ center is not involved. Electrochemical reduction is restricted to heme $a$ whereas electron transfer from heme $a$ to heme $a_{3}$, which in solution occurs on the nanosecond time scale, is drastically slowed down. It may be that the network of cooperativities that links intramolecular electron transfer and proton translocation is perturbed in the immobilised enzyme, possibly due to the effect of the interfacial electric field.

\section{Introduction}

Redox proteins immobilised on electrodes have been attracting the interest of researchers in both fundamental and applied science. ${ }^{1,2}$ Such devices promise to have a high potential in various fields of biotechnology including biocatalysis, biosensoring, or nanoelectronics. Furthermore, immobilised proteins offer several advantages for studying elementary biochemical and biophysical processes. Electron transfer reactions can be investigated without interferences of mediators or artificial redox labels using the electrode as the direct electron donor or acceptor.

Until the beginning of the nineties, such studies have been based on the direct protein adsorption on bare metal or semiconductor electrodes, ${ }^{3}$ which, however, frequently cause denaturation of the proteins. These difficulties can be overcome by introducing biocompatible coatings that cover the electrodes and enable protein immobilisation in a more "natural" environment. ${ }^{4}$ Such coatings, which are usually fabricated by the self-assembly of amphiphiles with appropriate tails and head groups, have been employed to immobilise a variety of soluble proteins via different modes of binding. Modern electrochemical methods allow determination of redox potentials and electron transfer kinetics and thus provide valuable insight into the reaction behaviour of the adsorbed proteins. ${ }^{5,6}$ More recently, also stationary and time-resolved surface enhanced resonance Raman spectroscopy (SERRS)

\footnotetext{
${ }^{a}$ Technische Universität Berlin, Institut für Chemie, Sekr. PC14, Straße des 17., Juni 135, D-10623 Berlin, Germany. E-mail: hildebrandt@chem.tu-berlin.de.E-mail:dh.murgida@tu-berlin.de

${ }^{b}$ Forschungszentrum Juelich, IBI-2: Biologische Strukturforschung, D-52425 Jülich, Germany
}

have been applied to redox proteins on coated Ag electrodes. ${ }^{7-16}$ This technique selectively probes the vibrational spectra of the cofactors solely of the immobilised proteins such that it is possible to monitor the molecular structures of the redox sites following changes of the electrode potential. SERRS has been widely used to analyse the redox process of soluble heme proteins on electrodes coated with self-assembled monolayers (SAM) of alkanethiol derivatives. ${ }^{7-16}$ These studies afforded new information on the electric field effect on the structure of the adsorbed proteins as well as on the thermodynamics, mechanism, and kinetics of the interfacial processes.

Up to now, only a few electrochemical studies on immobilised membrane proteins have been reported. ${ }^{17-19}$ Novel immobilisation strategies, however, now allow extending the investigation also to this class of proteins. A promising approach is based on tethering the solubilised protein via a histidine-tag (His-tag) to an electrode functionalised with nickel nitrilotriacetate (Ni-NTA) and subsequent replacement of the detergent by phospholipids to reconstitute a quasinatural membrane environment (Fig. 1). ${ }^{20,21}$ This strategy has been successfully used for immobilising cytochrome $c$ oxidase $(\mathrm{CcO})$ as demonstrated, inter alia, by surface enhanced infrared absorption spectroscopy and SERRS. ${ }^{21,22}$

In our previous study, ${ }^{22}$ we have shown that the two heme cofactors of $\mathrm{CcO}$, i.e., heme $a$ and heme $a_{3}$, that are visible in SERRS, exhibit vibrational spectra very similar to the resonance Raman spectra of the solubilised enzyme indicating that the structures of these redox sites are preserved in the immobilised state. Furthermore, potential-dependent SERRS reveals that electron transfer takes place between the electrode and the attached enzyme although a more detailed analysis was prohibited due to interference with photoreduction. Here, 


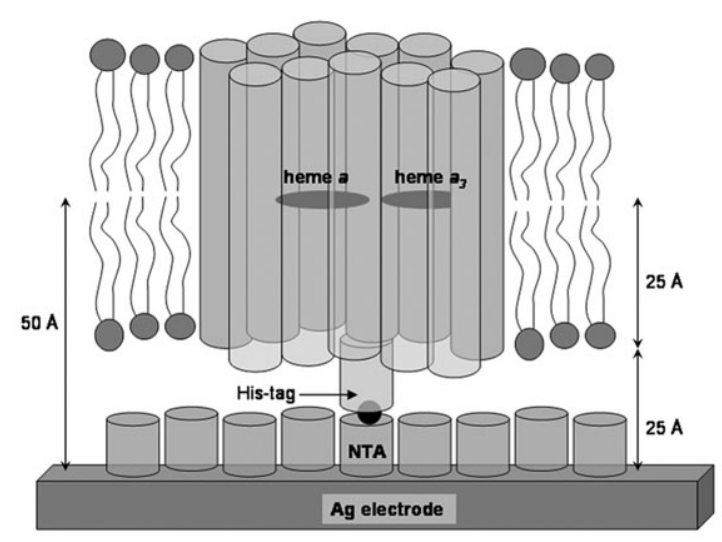

Fig. 1 Schematic presentation of $\mathrm{CcO}$ immobilised on an $\mathrm{Ag}$ electrode. The approximate positions of heme $a$ and heme $a_{3}$ in $\mathrm{CcO}$ (long rods) and distances to the electrode are estimated on the basis of the crystal structure. ${ }^{44}$ The small rods symbolize the NTA monolayer and the coordinating $\mathrm{Zn}^{2+}$ or $\mathrm{Ni}^{2+}$ ion is represented by the black circle.

we have used a novel experimental set-up by which photoreduction can be suppressed. Thus, a systematic investigation of the heterogeneous electron transfer processes of immobilised $\mathrm{CcO}$ is possible which is reported in this work.

\section{Materials and methods}

\section{Immobilisation of cytochrome $c$ oxidase}

The immobilisation procedure is based on the affinity of a Histagged $\mathrm{CcO}$ towards a metal ion chelating coating on the electrode. Two different protein variants of $\mathrm{CcO}$ from Rhodobacter sphaeroides, which carry the His-tag on the C-terminus either of subunit I (CcO-I) or of subunit II (CcO-II), have been used. The proteins have been expressed and purified as described elsewhere. ${ }^{23}$ For protein immobilisation, the electrochemically roughened $\mathrm{Ag}$ electrode was first immersed in a 1 $\mathrm{mM}$ aqueous solution of 3,3'-dithiobis[ $N$-(5-amino-5-carboxypentyl)propionamide- $N^{\prime}, N^{\prime}$-diacetic acid] (NTA) (Dojindo) for $c a .15 \mathrm{~h}$. Under these conditions, a densely packed SAM is formed on the metal surface via covalent linkage with the sulfur group as shown previously. ${ }^{20,21}$ Subsequently, the electrode was rinsed with water and immersed in an aqueous solution containing $50 \mathrm{mM}$ acetate buffer $(\mathrm{pH} \mathrm{5.5)}$ and either $40 \mathrm{mM} \mathrm{ZnCl} 2$ or $40 \mathrm{mM} \mathrm{NiCl}$. After $c a .1 \mathrm{~h}$ the electrode surface was rinsed with water and incubated in a phosphate buffer solution $(50 \mathrm{mM}, \mathrm{pH} 8,100 \mathrm{mM} \mathrm{KCl})$ containing 0.2 $\mu \mathrm{M} \mathrm{CcO}$ and $0.1 \% n$-dodecyl- $\beta$-D-maltoside (DM; Sigma) for $2 \mathrm{~h}$. For reconstitution of the lipid environment, the electrode surface was rinsed with buffer/DM solution to remove excess protein. Subsequently, the electrode was immersed in buffer/ DM solution containing additionally $0.05 \mathrm{mg} \mathrm{ml}^{-1}$ 1,2-diphytanoyl-sn-glycero-3-phosphocholine (Avanti Polar Lipids). Finally, the detergent was removed by addition of Bio-Beads (Bio-Rad) to the solution for $2 \mathrm{~h}$ upon stirring. ${ }^{24} \mathrm{In}$ this way, two different uniformly oriented monolayers of membranebound $\mathrm{CcO}$ could be obtained; one with the binding domain for its natural reaction partner cytochrome $c(\mathrm{Cyt}-c)$, i.e., the electron entry gate of $\mathrm{CcO}$, pointing to the solution phase
(CcO-I), and the other with the binding domain pointing to the electrode (CcO-II).

\section{Surface enhanced resonance Raman spectroscopy}

Surface enhanced resonance Raman (SERR) spectra were measured at ambient temperature with the $413 \mathrm{~nm}$ excitation line of a $\mathrm{Kr}^{+}$-laser (Coherent Innova 300) using a confocal Raman microscope (LabRam, HR800) equipped with a liquid nitrogen cooled CCD camera. The spectral resolution was 2 $\mathrm{cm}^{-1}$ and the wavenumber increment per pixel was $0.57 \mathrm{~cm}^{-1}$. The laser beam was focused on the surface of a disk $\mathrm{Ag}$ electrode $\left(0.9 \mathrm{~cm}^{2}\right)$ by means of a water immersion objective $(100 \times$, N. A. 1.00) with a working distance of $1.5 \mathrm{~mm}$. The $\mathrm{Ag}$ electrode was placed in a home-built electrochemical cell $(<2$ $\mathrm{mL}$ ) which includes a $\mathrm{Pt}$ wire and an $\mathrm{Ag} / \mathrm{AgCl}$ electrode as a counter and reference electrode, respectively. All potentials cited in this work refer to the normal hydrogen electrode. In contrast to the previously used $\mathrm{Ag}$ ring electrodes, ${ }^{11}$ a flat surface was found to be advantageous as it could be readily adapted to the short working distance of the microscope objective and allows an easier handling during the time-consuming multi-step sample preparation. The nylon cell body was mounted on an eccentrically rotating device such that the electrode surface is continuously moved with respect to the incident laser beam as a prerequisite for avoiding laser-induced photoreduction of the immobilised $\mathrm{CcO}$. In addition, a laser power as low as $100 \mu \mathrm{W}$ had to be used and the accumulation time was not more than $20 \mathrm{~s}$ (vide infra). To improve the signal-to-noise ratio, two or more spectra measured under the same experimental conditions but from different spots on the electrode were summed up. For measurements under anaerobic conditions, the cell was purged with catalytically purified Ar (Chromopack N) for $1 \mathrm{~h}$. During the experiments, a constant Ar overpressure was maintained by accommodating the cell into a home-built elastic plastic tent. In all SERRS experiments, the coated electrode was in contact with a solution containing $100 \mathrm{mM} \mathrm{KCl}$ and $50 \mathrm{mM}$ potassium phosphate $(\mathrm{pH} 8)$.

Resonance Raman spectra were measured with the equipment described previously using the rotating cell technique. ${ }^{10}$ The fully oxidized state of the enzyme was readily obtained under aerobic conditions whereas the fully reduced state was prepared by reduction with dithionite. The resonance Raman experiments were carried out with solutions containing 100 $\mathrm{mM} \mathrm{KCl}, 50 \mathrm{mM}$ potassium phosphate $(\mathrm{pH} 8), 25 \mu \mathrm{M} \mathrm{CcO}$ and $0.1 \% \mathrm{DM}$.

\section{Data analysis}

For the quantitative analysis of the SERR spectra measured as a function of the electrode potential, we have employed two strategies. In general, a manual background correction of the raw experimental spectra was carried out using a polynomial subtraction. Series of measurements that include a large number $(>40)$ of experimental spectra were also subjected to singular value decomposition (SVD) to determine the number of the main components. For those subspectra which are associated with the largest $S_{i i}$ values, the background was determined and subsequently subtracted from the original 
spectra according to the corresponding $\mathrm{V}_{i j}$ coefficients. This procedure was less time-consuming and largely avoids arbitrariness associated with the manual background subtraction. The background-corrected spectra obtained by both procedures were further analyzed by a band fitting procedure. A component analysis as employed in previous SERRS studies of heme proteins ${ }^{9-13}$ was not possible since a reliable determination of the four component spectra (i.e., reduced and oxidized hemes $a$ and $a_{3}$ ) was aggravated by the strong overlap of the porphyrin modes, particularly in the region between 1530 and $1650 \mathrm{~cm}^{-1}$. Thus, the quantitative analysis of the spectra was based on individual bands as described below.

\section{Results}

The resonance Raman spectra of heme proteins include a variety of bands that allow an unambiguous determination of the oxidation, spin, and coordination state of the heme. These marker bands, originating from the stretching modes of the porphyrin macrocycle, are found in the region between 1300 and $1700 \mathrm{~cm}^{-1}$ which, hence, displays a complex vibrational band pattern since $\mathrm{CcO}$ contains two hemes in two different spin and ligation states, i.e., a six-coordinated low spin (6cLS) and a six-coordinated high spin (6cHS) configuration in heme $a$ and heme $a_{3}$, respectively. ${ }^{25}$ Furthermore, the type- $a$ hemes of $\mathrm{CcO}$ include a conjugated vinyl and formyl substituent which give rise to resonance Raman-active stretching vibrations between 1610 and $1680 \mathrm{~cm}^{-1}$. The resonance Raman spectra of $\mathrm{CcO}$ from $R h$. spharoides have been analysed previously, ${ }^{26}$ and are similar to those of the mammalian and other bacterial enzymes. ${ }^{25-27}$

The most characteristic marker for the oxidation state originates from mode $\nu_{4}$, which is found at $c a .1370$ and $1358 \mathrm{~cm}^{-1}$ in the resonance Raman spectra of the fully reduced and fully oxidized state of $\mathrm{CcO}$, respectively (Fig. $2 \mathrm{~A}, \mathrm{E})$. These peaks represent the envelopes of the closely spaced bands of both hemes. However, heme $a$ and heme $a_{3}$ can be distinguished on the basis of the formyl stretching modes. The weakly hydrogen-bonded formyl substituent of heme $a_{3}$ gives rise to an isolated band at 1671 and $1663 \mathrm{~cm}^{-1}$ in the ferric and ferrous state, respectively. ${ }^{26,27}$ The substantially stronger hydrogen bonding interactions of the $\mathrm{C}=\mathrm{O}$ group in heme $a$ cause a distinct frequency downshift to 1646 and 1610 $\mathrm{cm}^{-1}$ in the oxidized and reduced form, respectively. A further unique marker band for the reduced heme $a$ is the wellseparated and relatively intense band at $1517 \mathrm{~cm}^{-1}\left(\nu_{11}\right)$. Both the porphyrin modes $\nu_{4}$ and $\nu_{11}$ and the formyl stretching modes allow monitoring the oxidation states of the individual hemes of the immobilised $\mathrm{CcO}$ and are therefore used for a quantitative analysis of the spectra by means of band fitting. Relative contributions of the reduced and oxidized hemes are then expressed in terms of relative intensities of the $\nu_{4}$ bands, which, however, systematically underestimates the relative concentrations of the reduced hemes for which the resonance enhancement at $413 \mathrm{~nm}$ is lower than for the oxidized hemes. ${ }^{22}$

\section{Photoreduction}

Fig. 2B, C, and D display SERR spectra of CcO-I measured at open circuit under different irradiation conditions. At a laser

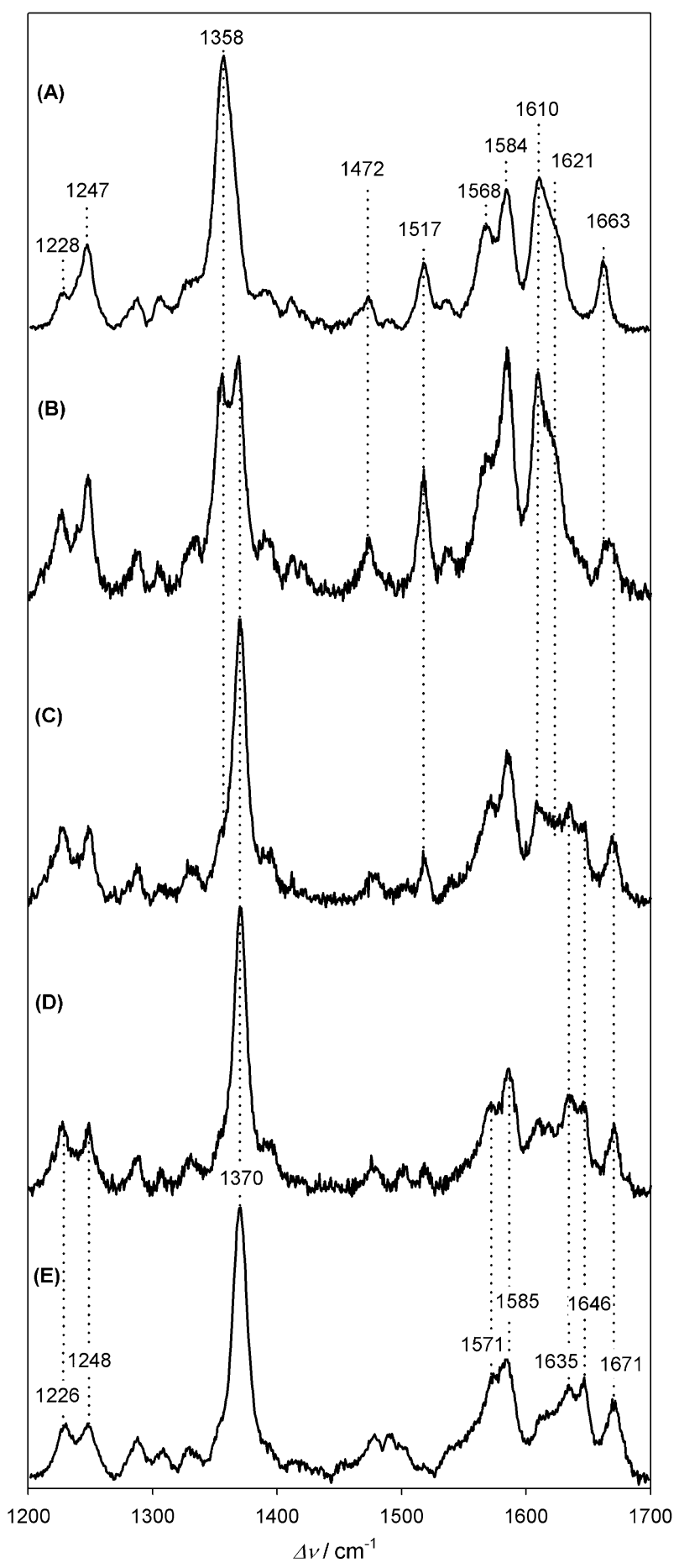

Fig. 2 Resonance Raman spectra of (A) fully reduced and (E) fully oxidised $\mathrm{CcO}$ in solution compared with $(\mathrm{B}, \mathrm{C}, \mathrm{D})$ SERR spectra of immobilised $\mathrm{CcO}$ at open circuit. All spectra were measured with 413$\mathrm{nm}$ excitation. Resonance Raman spectra were obtained from the sample in a rotating cell with a laser power of $10 \mathrm{~mW}$ using the set-up described previously. ${ }^{10}$ The SERR spectra were measured with a confocal arrangement (see Materials and Methods) using different excitation conditions: $\mathrm{B}, 0.1 \mathrm{~mW}$, stationary electrode; C, $0.3 \mathrm{~mW}$, rotating electrode; $\mathrm{D}, 0.1 \mathrm{~mW}$, rotating electrode. 
power of $0.1 \mathrm{~mW}$ focused onto the same spot of the surface (i.e., no rotation of the cell; Fig. 2B), the portion of reduced hemes is relatively high as indicated by the intensity ratio of the $\nu_{4}$ modes. Since also the $\nu_{11}$ mode at $1517 \mathrm{~cm}^{-1}$ is very intense and the $\mathrm{C}=\mathrm{O}$ stretching at $1646 \mathrm{~cm}^{-1}$ is not detectable, we conclude that it is mainly heme $a$ that is reduced whereas heme $a_{3}$ appears to be in a mixture of oxidized and reduced form since the $\mathrm{C}=\mathrm{O}$ stretching region of heme $a_{3}$ displays the corresponding bands at 1671 and $1663 \mathrm{~cm}^{-1}$ with nearly equal intensity. Moving the electrode surface with respect to the laser beam via eccentric rotation of the cell drastically lowers reduction such that the spectrum measured with an even higher laser power $(0.3 \mathrm{~mW})$ reflects only a small amount of reduced heme (Fig. 2C). As judged from the still relatively strong $1517-\mathrm{cm}^{-1}$ band and the intensity increase at $1610 \mathrm{~cm}^{-1}$, reduction should be largely restricted to heme $a$. A further lowering of the laser exposure by using a laser power of $0.1 \mathrm{~mW}$, causes the disappearance of the 1358 - and $1517-\mathrm{cm}^{-1}$ bands indicating that the immobilised $\mathrm{CcO}$ is essentially in the fully oxidized form. These findings demonstrate that the enzyme can easily be photoreduced by the incident laser beam. On the other hand, direct electron transfer from the electrode does not take place under these conditions although the formal potential of the working electrode at open circuit corresponds to $c a$. $+0.27 \mathrm{~V}$, which lies between the redox potentials of heme $a(+0.39 \mathrm{~V})$ and heme $a_{3}(+0.2 \mathrm{~V})$ as determined for the fully oxidized enzyme. ${ }^{28}$

\section{Different modes of $\mathrm{CcO}$ immobilisation}

Immobilsation of CcO-I and CcO-II leads to the same SERR spectra. Mutual subtraction of the SERR spectra of CcO-I and $\mathrm{CcO}-\mathrm{II}$ measured at the same potential and under identical conditions yields a difference spectrum with no negative or positive peaks. Thus, we conclude that the surface enhancement of the resonance Raman scattering is about the same for $\mathrm{CcO}$ binding via the $\mathrm{C}$-terminus of subunit I or II. This finding can be understood since both modes of $\mathrm{CcO}$ binding correspond to approximately the same distances and orientations of the hemes with respect to the metal surface (Fig. 1). Also the exchange of $\mathrm{Ni}^{2+}$ by $\mathrm{Zn}^{2+}$ as the coordinating metal ion for the protein bound His-tag does not affect the SERR spectra in the potential range between +0.2 and $-0.4 \mathrm{~V}$ and has no detectable influence on the redox behaviour. However, the $\mathrm{Zn}$ NTA complex is electrochemically inert even at more negative potentials, ${ }^{29,30}$ such that Zn-NTA was used specifically for potential-dependent measurements of $\mathrm{CcO}$ under anaerobic conditions at which the potential range was extended below $-0.4 \mathrm{~V}$.

\section{Reduction of $\mathrm{CcO}$ via heterogeneous electron transfer under anaerobic conditions}

SERR spectra were measured after careful degassing the solution with Ar and upon maintaining an Ar overpressure in the rotating electrochemical cell during the measurements. The irradiation conditions $(0.1 \mathrm{~mW}$, rotating cell) were chosen such that photoreduction was avoided. Under these conditions, the SERR spectrum measured at $+0.2 \mathrm{~V}$ displays the signature of a nearly fully oxidized $\mathrm{CcO}$ (Fig. 3). A band fitting

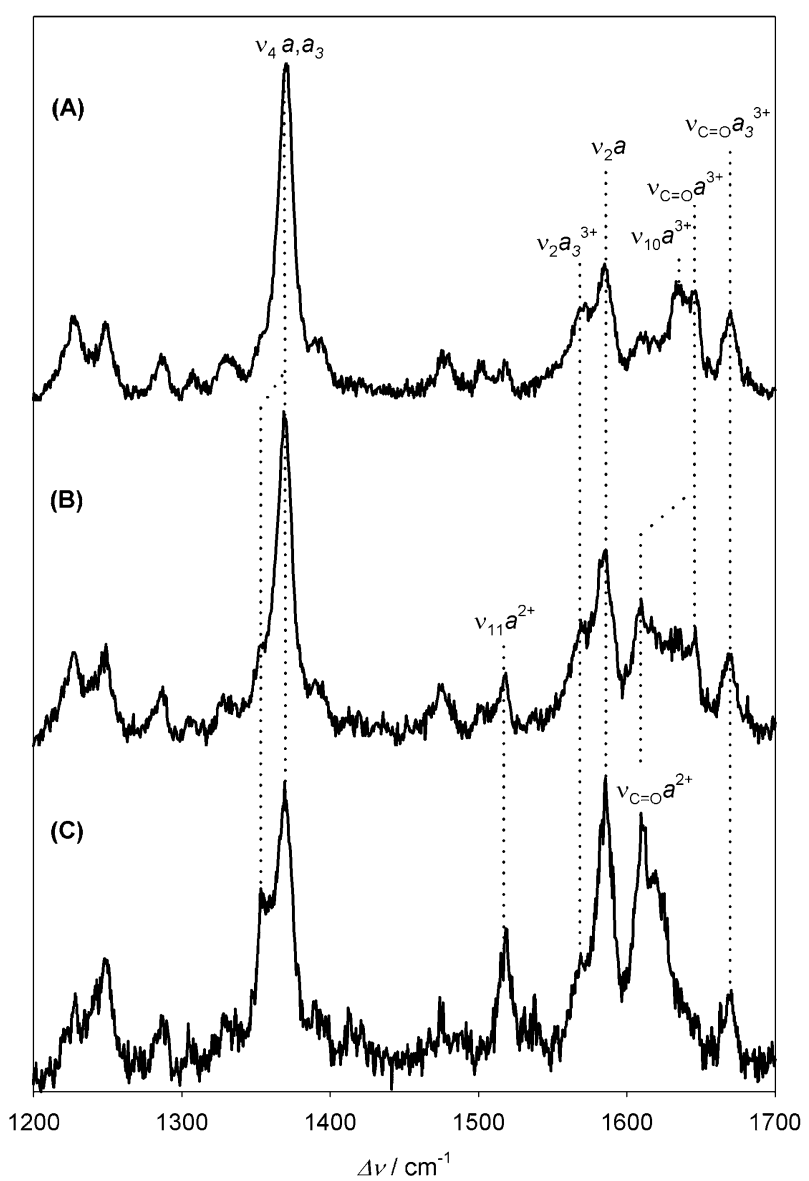

Fig. 3 SERR spectra of immobilised $\mathrm{CcO}$ using a rotating electrode and a laser power of $0.1 \mathrm{~mW}(413 \mathrm{~nm})$ under anaerobic conditions but at different potentials: $\mathrm{A},+0.2 \mathrm{~V}, \mathrm{~B},-0.1 \mathrm{~V}, \mathrm{C},-0.45 \mathrm{~V}$.

analysis of the $\nu_{4}$ envelope reveals a spectral contribution of the reduced forms of less than $5 \%$. Decreasing the potential leads to a growing-in of the reduced form to $22 \%$ at $-0.2 \mathrm{~V}$ and $39 \%$ at $-0.45 \mathrm{~V}$ (Fig. $4 \mathrm{a}$ ). Whereas the analysis of the $\nu_{4}$ envelope only provides information about the total amount of reduced hemes, the inspection of the formyl stretching region indicates that it is primarily heme $a$ that is reduced with decreasing potential since the $1671-\mathrm{cm}^{-1}$ band remains largely unchanged whereas the $1646-\mathrm{cm}^{-1}$ gradually disappears. The lack of the $1646-\mathrm{cm}^{-1}$ band at $-0.45 \mathrm{~V}$ implies that at this potential heme $a$ is essentially completely reduced. This conclusion is in line with the intensity increase of the $1517-\mathrm{cm}^{-1}$ band ( $\nu_{11}$ of ferrous heme $a$ ) that accompanies the growing-in of the band at $1358 \mathrm{~cm}^{-1}$.

To estimate the rate of the electron transfer from the electrode to heme $a$, the electrode potential was changed from open circuit $(+0.27 \mathrm{~V})$ to $0.0 \mathrm{~V}$ and the SERR spectra were measured at different delay times with respect to the potential jump. The quantitative analysis of the SERR spectra reveals a time-dependent reduction that can be approximated by a single exponential function corresponding to a rate constant of $(0.002 \pm 0.0005) \mathrm{s}^{-1}$ (Fig. 4b). The subsequent potential jump to $-0.2 \mathrm{~V}$ leads to a further increase of the reduced form 

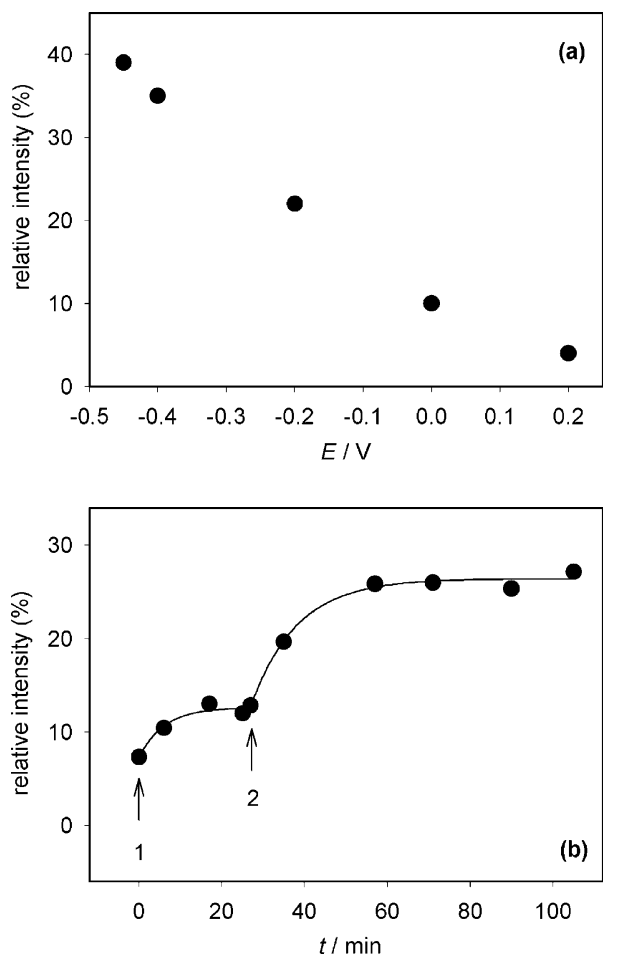

Fig. 4 Redox equilibria and kinetics of immobilised $\mathrm{CcO}$ under anaerobic conditions determined from the $\nu_{4}$ band intensities of the SERR spectra: (a), equilibrium spectral contributions of the reduced hemes as a function of the electrode potential; (b), changes of the spectral contributions of the reduced hemes following a potential jump from (1) open circuit $(c a .+0.27 \mathrm{~V})$ to $0.0 \mathrm{~V}$, and from (2) $0.0 \mathrm{~V}$ to $-0.2 \mathrm{~V}$. Further details are given in the text.

albeit with the same rate $\left(0.002 \mathrm{~s}^{-1}\right)$. Experiments with CcO-I and $\mathrm{CcO}-\mathrm{II}$ yield essentially the same results both with respect to the degree and the rate of reduction.

Whereas up to $c a$. $-0.45 \mathrm{~V}$, reduction appears to be largely restricted to heme $a$, a further decrease of the electrode potential leads to a steady increase of the contribution of the ferrous heme $a_{3}$ (Fig. 5). The underlying spectral changes, however, point to further chemical processes in addition to the reduction of this cofactor. Specifically, we note a substantial intensity decrease of the $\nu_{4}$ bands and of the heme $a_{3}$ formyl stretching relative to the bands at 1247,1584 , and $1610 \mathrm{~cm}^{-1}$, which is distinctly different compared to the resonance Raman spectrum of the fully reduced enzyme in solution.

\section{Reduction of $\mathrm{CcO}$ via heterogeneous electron transfer under aerobic conditions}

Potential-dependent SERRS measurements of CcO-I and $\mathrm{CcO}-\mathrm{II}$ in the presence of oxygen reveal a qualitatively similar picture as under anaerobic conditions since the only reduced component that is formed is ferrous heme $a$. Reduction of heme $a$ following a potential jump from open circuit to $-0.2 \mathrm{~V}$ and subsequently to $-0.4 \mathrm{~V}$ occurs with slightly slower rate constants than under anaerobic conditions (data not shown).

Cyanide binding to solubilised $\mathrm{CcO}$ has been extensively studied previously using UV-Vis absorption as well as IR and

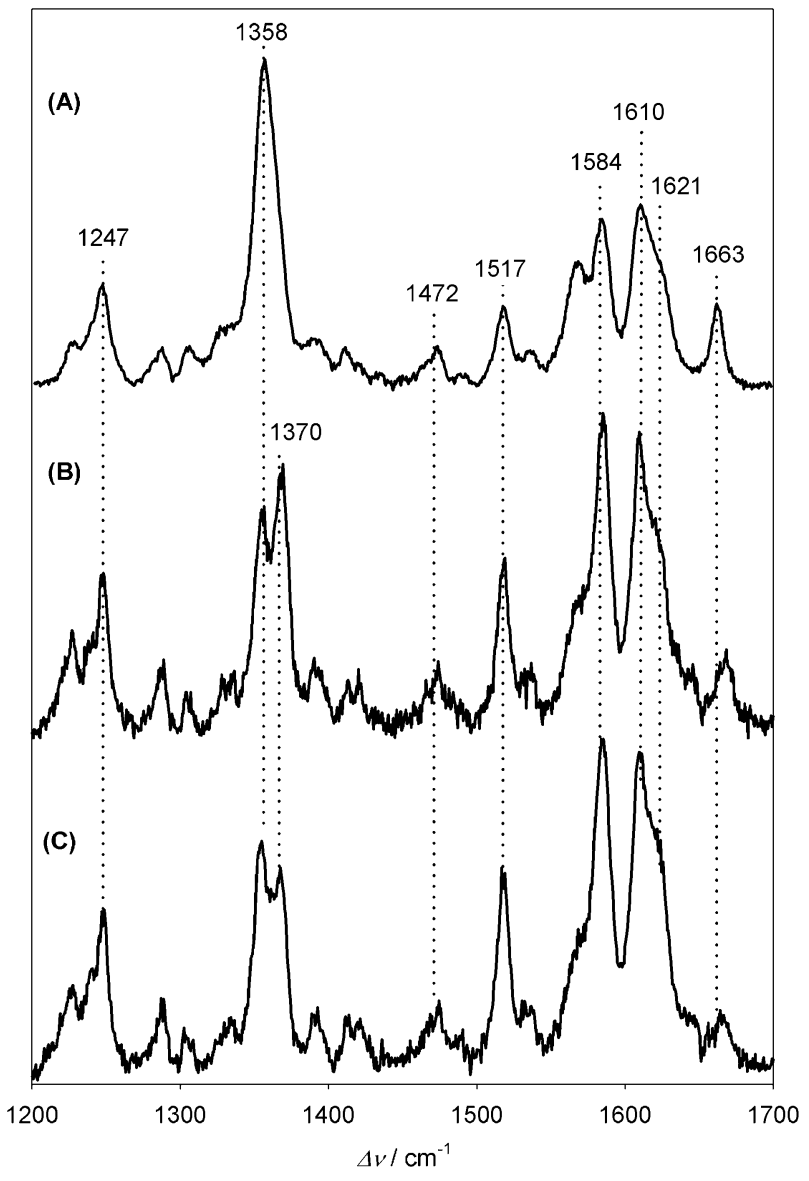

Fig. 5 SERR spectra of immobilised $\mathrm{CcO}$ using a rotating electrode and a laser power of $0.1 \mathrm{~mW}(413 \mathrm{~nm})$ under anaerobic conditions at (B) $-0.8 \mathrm{~V}$ and (C) $-1.1 \mathrm{~V}$, compared with (A) the resonance Raman spectrum of the fully reduced $\mathrm{CcO}$ in solution (see Fig. 2).

resonance Raman spectroscopy. ${ }^{31-39}$ To immobilise the fully oxidised cyanide complex of $\mathrm{CcO}(\mathrm{CcO}[\mathrm{CN}])$, the enzyme was incubated with a 200 -fold excess of cyanide for $1 \mathrm{~h}$ prior to immobilisation. Also during the individual steps of the immobilisation procedure, a 200 -fold excess of cyanide was maintained. Thus, it is possible that cyanide is co-adsorbed on the electrode surface which would account for the decrease of the open circuit potential to $+0.05 \mathrm{~V}$. UV-Vis absorption spectra confirmed the formation of $\mathrm{CcO}[\mathrm{CN}]$ since the Soret maximum of the fully oxidised form shifts from $421 \mathrm{~nm}$ to 428 $\mathrm{nm}$ after $\mathrm{CN}$ addition. Taking into account that cyanidebinding to heme $a_{3}$ causes a transition from the $6 \mathrm{cHS}$ to the 6cLS configuration, the changes in the SERR spectra are only small (data not shown). However, they are in line with previous findings for solubilised $\mathrm{CcO}{ }^{33}$ The changes mainly refer to the intensity increase at $1585 \mathrm{~cm}^{-1}$ at the expense of the $1570-\mathrm{cm}^{-1}$ band, reflecting the expected $c a \cdot 15-\mathrm{cm}^{-1}$ frequency upshift associated with the spin state change. On the other hand, the formyl stretching and the $\nu_{4}$ band as well as the heme $a$ modes remain unaffected such that the same marker bands can be used for probing the redox transition as in the case of the cyanide-free enzyme. 

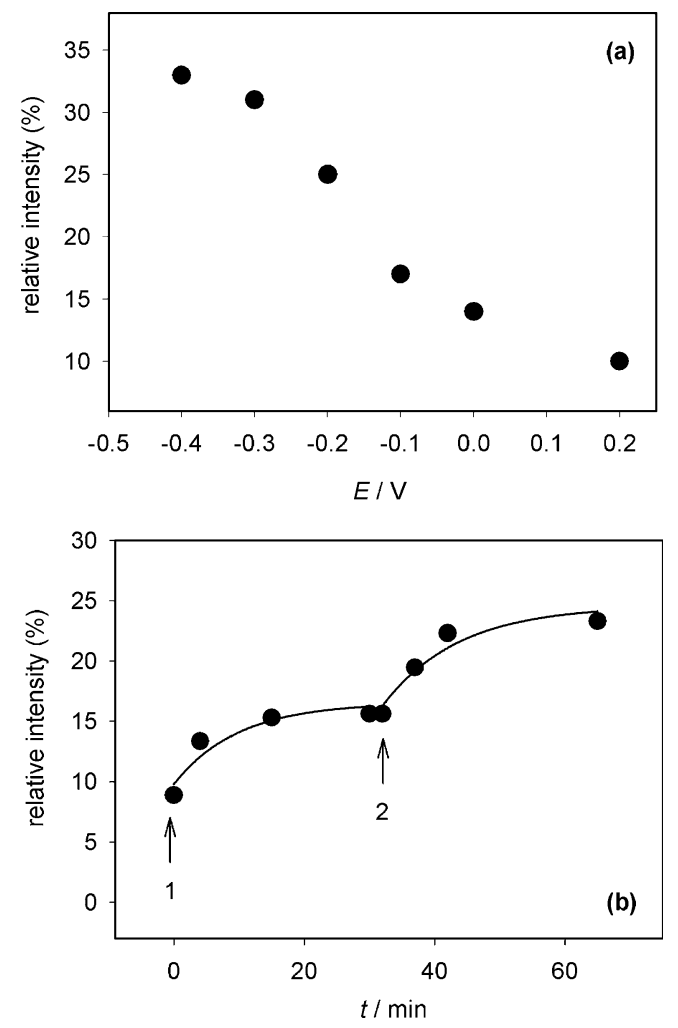

Fig. 6 Redox equilibria and kinetics of immobilised cyanide complex of $\mathrm{CcO}$ under aerobic conditions determined from the $\nu_{4}$ band intensities of the SERR spectra. (a), equilibrium spectral contributions of the reduced hemes as a function of the electrode potential; (b), changes of the spectral contributions of the reduced hemes following a potential jump from (1) open circuit $(c a$. $+0.05 \mathrm{~V})$ to $-0.1 \mathrm{~V}$, and from (2) $-0.1 \mathrm{~V}$ to $-0.2 \mathrm{~V}$. Further details are given in the text.

The SERR spectra of $\mathrm{CcO}[\mathrm{CN}]$ measured under non-photoreductive conditions reflect an increasing reduction upon lowering the potential as indicated by the intensity re-distribution of the $\nu_{4}$ envelope. The concomitant intensity increase of the $1517-\mathrm{cm}^{-1}$ band and the preservation of the formyl stretching at $1671 \mathrm{~cm}^{-1}$ imply that reduction is largely restricted to heme $a$. At $-0.4 \mathrm{~V}$, the total portion of reduced hemes, expressed in terms of the $\nu_{4}$ intensities, appears to reach a maximum value with $c a .35 \%$ which as well as the rate of reduction at $-0.1 \mathrm{~V}$ and $-0.2 \mathrm{~V}\left(0.002 \mathrm{~s}^{-1}\right)$ (Fig. 6) is comparable to that found for $\mathrm{CcO}$ under anaerobic conditions. Again no differences were found for the cyanide-complexes of CcO-I and CcO-II.

\section{Discussion}

The present results have shown that membrane bound $\mathrm{CcO}$ can be immobilised on an $\mathrm{Ag}$ electrode under preservation of the native active site structure in the potential range between +0.3 and $-0.4 \mathrm{~V}$, thereby confirming previous observations. ${ }^{22}$ Either Ni-NTA- or Zn-NTA-functionalised surfaces may serve to attach the His-tagged enzyme including a lipid bilayer environment to the electrode. However, the reaction behaviour of the immobilised $\mathrm{CcO}$ is quite different compared to the solubilised enzyme in solution.

\section{Photoreduction and heme degradation}

Photoreduction of heme proteins and specifically of $\mathrm{CcO}$ in solution has been reported previously. ${ }^{32,40-42}$ In the SERRS experiment, this process is likely to be more efficient than in solution since the interactions of the radiation field with the surface plasmons of the metal do not only enhance the resonance Raman scattering but also all other photophysical processes. ${ }^{43}$ The increase of the laser power from $0.1 \mathrm{~mW}$ (no photoreduction) to $0.3 \mathrm{~mW}$ causes an acceleration of the heme $a$ reduction rate by $c a$. one order of magnitude. The enhanced rate of reduction under photoreductive conditions depends on the quantum yield of the primary photoprocess and possibly also on the location of the photoreducible site in the protein if its distance to the hemes is large. Also photoreduction occurs stepwise with heme $a$ being the first heme to be reduced, followed by heme $a_{3}$.

Up to now, the mechanism of photoreduction is not known; however, the present data suggest that it is associated with a chemical modification of the hemes. Whereas the SERR spectrum and the resonance Raman spectrum of fully oxidized $\mathrm{CcO}$ (Fig. 2D, E) agree very well with respect to frequencies and relative intensities, the SERR spectrum measured under strong photoreduction (Fig. 2B) cannot be represented as the sum of the resonance Raman spectra of the fully reduced and fully oxidized enzyme. Specifically, we note a drastic decrease of the $\nu_{4}$ mode intensities with respect to most of the other bands. These spectral changes are similar to those observed under non-photoreductive conditions at very negative electrode potentials (Fig. 5). In that case, the tetrapyrrole macrocycles may be attacked by reactive oxygen species that are intermediary formed after direct electrochemical reduction of molecular oxygen which is inevitably present in traces even under "anaerobic" conditions. Also photoreduction may be associated with the formation of reactive intermediates that are capable to attack the porphyrins. These species may be generated via reaction of oxygen or water with the "electron hole" at the photooxidised site.

\section{Long distance electron hopping}

A notable reduction of the immobilised enzyme requires electrode potentials that are negative compared to the redox potential of the cofactors. Due to the relatively large separation of the redox sites from the electrode and specifically the solution phase between the NTA monolayer and the tethered protein, the true potential at the cofactor sites is likely to be substantially different from that poised at the working electrode. ${ }^{10}$ Thus, it is not straightforward to relate the difference between electrode potential and the redox potential to the driving force for the electron transfer.

At electrode potentials between 0.0 and $-0.4 \mathrm{~V}$, heterogeneous electron transfer from the electrode to heme $a$ occurs with a rate constant of $c a .0 .002 \mathrm{~s}^{-1}$ under anaerobic conditions and in the presence of cyanide. Moreover, these rate constants are approximately the same for $\mathrm{CcO}$ attachment via the His-tag on the C-terminus of either subunit I or subunit II. This finding implies that immobilisation of $\mathrm{CcO}$ with the natural electron entry gate close to the electrode, i.e., CcOII, does not facilitate the heterogeneous electron transfer. 
Consequently, we conclude that the primary electron acceptor, the $\mathrm{Cu}_{\mathrm{A}}$ site, ${ }^{44}$ which is not detectable for SERRS, is not required for the reduction of the heme sites of the immobilised enzyme and electron transfer occurs via an alternative pathway through the protein.

Both heme $a$ and heme $a_{3}$ are located in the center of the transmembrane helices with quite similar distances to both sides of the membrane. Accordingly, the through space distances from the electrode to the heme groups are $c a$. $50 \AA$ for both orientations of the enzyme (CcO-I and CcO-II). For such large distances electron tunnelling is highly unlikely and cannot account for the experimentally determined rate constants for the reduction of heme $a{ }^{43,45}$ Instead, it is concluded that electron transfer occurs via an electron hopping mechanism which at electron transfer distances longer than $30 \AA$ may effectively compete with electron tunnelling. In fact, Morita and Kimura have recently demonstrated electron hopping through helical peptides self-assembled on Au electrodes. In that study, rate constants of $c a .1 \mathrm{~s}^{-1}$ were determined for an electron transfer distance of more than $40 \AA$ that are not consistent with an electron tunnelling process. ${ }^{46}$ For CcO-I and $\mathrm{CcO}-\mathrm{II}$, the electron transfer distance is even larger by at least $10 \AA$ which, in the case of electron tunnelling, would correspond to an electron transfer rate constant lower than $10^{-10} \mathrm{~s}^{-1},{ }^{11}$ and thus cannot be reconciled with the experimentally observed value of $c a .0 .002 \mathrm{~s}^{-1}$. Furthermore, no distinct dependence on the overpotential is observed for the heterogeneous electron transfer to $\mathrm{CcO}$ which also supports an electron hopping mechanism according to theoretical predictions, ${ }^{45}$ and the experimental results for immobilised peptides. ${ }^{46}$ In the latter case, Morita and Kimura suggested the amide groups of the peptide chain to be the hopping sites. ${ }^{46}$ This conclusion may also be valid for the immobilised $\mathrm{CcO}$ although the coordinating metal center of the His-tag may be involved as well. However, we did not note a difference in the electron transfer dynamics for Ni-NTA- and Zn-NTA-functionalised electrodes.

\section{Selective reduction of heme $a$}

Although the electron transfer distances to hemes $a$ and $a_{3}$ are nearly the same, only heme $a$ is electrochemically reduced at potentials above $-0.45 \mathrm{~V}$. This result is even more surprising since for electron transfer reactions of the solubilised enzyme a rapid intramolecular electron transfer from heme $a$ to heme $a_{3}$ is found. ${ }^{47}$ In the fully oxidised state, heme $a$ exhibits a significantly more positive redox potential $(+0.39 \mathrm{~V})$ than heme $a_{3}(+0.2 \mathrm{~V}),{ }^{28}$ which would correspond to an uphill electron transfer. However, upon reduction of heme $a_{3}$, the redox potentials are nearly reversed which has been attributed to a negative cooperativity. ${ }^{48-50}$ This effect refers to the coupling of the energy levels of the electron and a proton via a structural change in the protein which is ultimately linked to the translocation of protons concomitant to electron transfer. Following this interpretation, we conclude that the anti-cooperativity between the heme $a$ and $a_{3}$ redox sites is efficiently blocked for the immobilised enzyme on the electrode such that electron transfer from heme $a$ to heme $a_{3}$ becomes even slower than the heterogeneous electron transfer from the electrode to heme $a$. Then, once heme $a_{3}$ is reduced, this step is likely to be followed immediately by binding of oxygen which is present in the solution and in the membrane in traces also under anaerobic conditions. The oxygen-bound heme $a_{3}$ as well as the subsequent intermediates are not expected to contribute to the SERR spectrum since their kinetics are much faster than heterogeneous electron transfer. ${ }^{51}$ Thus, the only species that are observed under these steady state conditions are the fully oxidised enzyme and the mixed-valence state involving the reduced heme $a$ and the oxidised heme $a_{3}$.

\section{The loss of anti-cooperativity in immobilised $\mathrm{CcO}$}

The present immobilisation strategy for $\mathrm{CcO}$ leaves the enzyme within an intact phospholipid bilayer which is expected to mimic the natural membrane environment more closely than the detergents used in the studies of the solubilised enzyme in solution. However, the immobilised enzyme appears to lack an essential feature, the cooperative couplings between heme $a$ and $a_{3}$, which under physiological conditions guarantees the energy transduction in $\mathrm{CcO}$.

SERRS probes the structure of the heme $a$ and $a_{3}$ sites and does not reveal any structural changes that would account for a perturbation of the electron flow between both hemes. Specifically, we note that the native hydrogen bond interactions of the formyl substituents of both heme groups with the protein environment appear to be preserved as indicated by the characteristic frequencies. There is also no change of the ligation state to a five-coordinated high spin ( $5 \mathrm{cHS}$ ) configuration form which is frequently observed for heme proteins immobilised on electrodes. ${ }^{8}$ In particular, the bridging ligand of heme $a_{3}$ sites of oxidases is only weakly bound such that in some cases a partial transition to the $5 \mathrm{cHS}$ form has been observed even in solution. ${ }^{52}$ For immobilised CcO-I and CcOII none of these structural changes has been detected such that the loss of anti-cooperativity cannot be related to a distortion of the heme site structures.

Unlike in the solution, electron and proton translocation of the immobilised $\mathrm{CcO}$ occurs in an electric field which may affect the mechanism and dynamics of charge translocation as well as protonation equilibria in proteins. ${ }^{8,53}$ Already subtle $\mathrm{p} K_{\mathrm{A}}$ changes of those protonable sites that are involved in the redox-linked proton translocation may alter substantially the network of cooperativities which ensures the functioning of the enzyme. ${ }^{49,50}$ In this respect, it is tempting to relate the present findings to those data obtained for $\mathrm{CcO}$ incorporated in phospholipids vesicles in a unidirectional manner, ${ }^{54-57}$ since local electric field strengths at electrodes and phospholipid bilayers may be comparable. ${ }^{8,58}$ It has been demonstrated that increasing the transmembrane potentials can efficiently block the enzymatic turnover due to a drastic slow down of proton or electron transfer or both, which in view of the present data may be understood in terms of electric-field induced $\mathrm{p} K_{\mathrm{A}}$ changes.

A completely different behaviour has been observed for the quinol oxidase from Acidianus ambivalens. ${ }^{59}$ In that case, the SERRS studies revealed the complete reduction of both heme $a$ and heme $a_{3}$ without any indication for a hindrance of the electron transfer between both cofactors. Moreover, 
both hemes display an ideal Nernstian behaviour with redox potentials in the reversed order as compared to $\mathrm{CcO}$, implying that internal electron transfer does not require a cooperative coupling between protonable and redox sites.

\section{Acknowledgements}

The work was supported by the Deutsche Forschungsgemeinschaft (Sfb 498; J. Hrabakova, P. Hildebrandt, D. H. Murgida) and by the Volkswagen-Stiftung (J. Heberle).

\section{References}

1 I. Willner and E. Katz, Angew. Chem., Int. Ed., 2000, 39, 1180.

2 J. W. Choi, Y. S. Nam and W. H. Lee, Curr. Appl. Phys., 2002, 2 , 79

3 E. F. Bowden, F. M. Hawkridge and H. N. Blount, in Comprehensive treatise of electrochemistry, ed. S. Srinivasan, Yu. A. Chiznadzhiev, J. O. M. Bockris, B. E. Conway and E. Yeager, Plenum Press, New York, 1985, ch. 5.

4 A. Ulman, in Introduction to Ultrathin Organic Films. From Langmuir-Blodgett to Self-Assembly, Academic Press, Boston, 1991, 279.

5 F. A. Armstrong and G. S. Wilson, Electrochim. Acta, 2000, 45, 2623.

6 E. P. Friis, J. E. T. Andersen, Y. I. Kharkats, A. M. Kuznetsov, R. J. Nichols, J. D. Zhang and J. Ulstrup, Proc. Natl. Acad. Sci. U. S. A., 1999, 96, 1379.

7 D. Hobara, K. Niki and T. M. Cotton, Biospectroscopy, 1998, 4, 161.

8 D. H. Murgida and P. Hildebrandt, Acc. Chem. Res., 2004, 37, 854.

9 D. H. Murgida and P. Hildebrandt, Angew. Chem., Int. Ed., 2001, 40, 728.

10 D. H. Murgida and P. Hildebrandt, J. Phys. Chem. B, 2001, 105, 1578 .

11 D. H. Murgida and P. Hildebrandt, J. Am. Chem. Soc., 2001, 123, 4062.

12 L. Rivas, D. H. Murgida and P. Hildebrandt, J. Phys. Chem. B, 2002, 106, 4823.

13 D. H. Murgida and P. Hildebrandt, J. Phys. Chem. B, 2002, 106, 12814.

14 L. A. Dick, A. J. Haes and R. P. Van Duyne, J. Phys. Chem. B, 2000, 104, 11752.

15 S. Bernad, T. Soulimane and S. Lecomte, J. Raman Spectrosc., 2004, 35, 47.

16 L. Rivas, C. M. Soares, A. M. Baptista, J. Simaan, R. DiPaolo, D. H. Murgida and P. Hildebrandt, Biophys. J., 2005, 88, 4188.

17 J. D. Burgess, M. C. Rhoten and F. M. Hawkridge, Langmuir, 1998, 14, 2467.

18 A. S. Haas, D. L. Pilloud, K. S. Reddy, G. T. Babcock, C. C. Moser, J. K. Blasie and P. L. Dutton, J. Phys. Chem. B, 2001, 105, 11351.

19 L. Y. Su, F. M. Hawkridge and M. C. Rhoten, Chem. Biodiversity, 2004, 1, 1281

20 F. Giess, M. G. Friedrich, J. Heberle, R. Naumann and W. Knoll, Biophys. J., 2004, 87, 3213.

21 K. Ataka, F. Giess, W. Knoll, R. Naumann, S. Haber-Pohlmeier, B. Richter and J. Heberle, J. Am. Chem. Soc., 2004, 126, 16199.

22 M. G. Friedrich, F. Giess, R. Naumann, W. Knoll, K. Ataka, J. Heberle, J. Hrabakova, D. H. Murgida and P. Hildebrandt, Chem. Commun., 2004, 2376.

23 C. Hiser, D. A. Mills, M. Schall and S. Ferguson-Miller, Biochemistry, 2001, 40, 1606.
24 J. L. Rigaud, G. Mosser, J. J. Lacapere, A. Olofsson, D. Levy and J. L. Ranck, J. Struct. Biol., 1997, 118, 226.

25 G. T. Babcock, in Biological Applications of Raman spectroscopy, ed. T. G. Spiro, Wiley, New York, 1988.

26 H. M. Lee, T. K. Das, D. L. Rousseau, D. Mills, S. FergusonMiller and R. B. Gennis, Biochemistry, 2000, 39, 2989.

27 G. E. Heibel, P. Hildebrandt, B. Ludwig, P. Steinrucke, T. Soulimane and G. Buse, Biochemistry, 1993, 32, 10866.

28 M. I. Verkhovsky, J. E. Morgan and M. Wikstrom, Biochemistry, 1995, 34, 7483.

29 D. L. Johnson, S. W. Polyak, J. C. Wallace and L. L. Martin, Lett. Peptide Sci., 2003, 10, 495.

30 D. L. Johnson and L. L. Martin, J. Am. Chem. Soc., 2005, 127, 2018.

31 T. Ogura, N. Sone, K. Tagawa and T. Kitagawa, Biochemistry, 1984, 23, 2826.

32 T. Ogura, S. Yoshikawa and T. Kitagawa, Biochemistry, 1985, 24, 7746.

33 Y. C. Ching, P. V. Argade and D. L. Rousseau, Biochemistry, 1985, 24, 4938.

34 S. R. Lynch, R. H. Carter and R. A. Copeland, Biochemistry, 1993, 32, 6923.

35 P. A. Harmon, R. W. Hendler and I. W. Levin, Biochemistry, 1994, 33, 699.

36 S. Hirota, T. Ogura, K. ShinzawaItoh, S. Yoshikawa and T. Kitagawa, J. Phys. Chem., 1996, 100, 15274.

37 E. Pinakoulaki, M. Vamvouka and C. Varotsis, J. Phys. Chem. B, 2003, 107, 9865.

38 E. Pinakoulaki, M. Vamvouka and C. Varotsis, Inorg. Chem., 2004, 43, 4907.

39 T. K. Das, C. M. Gomes, T. M. Bandeiras, M. M. Pereira, M. Teixeira and D. L. Rousseau, Biochim. Biophys. Acta, 2004, 1655, 306.

40 T. Masuda, A. Minemura, K. Yamauchi and M. Kondo, J. Radiat. Res., 1980, 21, 149.

41 Y. G. Gu, P. S. Li, J. T. Sage and P. M. Champion, J. Am. Chem. Soc., 1993, 115, 4993.

42 F. Adar and M. Erecinska, Biochemistry, 1979, 18, 1825.

43 R. A. Marcus and N. Sutin, Biochim. Biophys. Acta, 1985, 811, 265.

44 M. Svensson-Ek, J. Abramson, G. Larsson, S. Tornroth, P. Brzezinski and S. Iwata, J. Mol. Biol., 2002, 321, 329.

45 E. G. Petrov, Y. V. Shevchenko, V. I. Teslenko and V. May, J. Chem. Phys., 2001, 115, 7107.

46 T. Morita and S. Kimura, J. Am. Chem. Soc., 2003, 125, 8732.

47 B. C. Hill, J. Biol. Chem., 1991, 266, 2219.

48 M. Brunori, A. Giuffre, E. Ditri and P. Sarti, J. Biol. Chem., 1997, 272, 19870.

49 A. V. Xavier, FEBS Lett., 2002, 532, 261.

50 A. V. Xavier, Biochim. Biophys. Acta, 2004, 1658, 23.

51 G. T. Babcock, J. M. Jean, L. N. Johnston, G. Palmer and W. H. Woodruff, J. Am. Chem. Soc., 1984, 106, 8305.

52 S. Gerscher, P. Hildebrandt, G. Buse and T. Soulimane, Biospectroscopy, 1999, 5, S53.

53 E. Neumann, Prog. Biophys. Mol. Biol., 1986, 47, 197.

54 P. M. Moroney, T. A. Scholes and P. C. Hinkle, Biochemistry, 1984, 23, 4991.

55 L. Gregory and S. Fergusonmiller, Biochemistry, 1989, 28, 2655.

56 P. Sarti, G. Antonini, F. Malatesta and M. Brunori, Biochem. J., 1992, 284, 123.

57 P. Nicholls and P. Butko, J. Bioenerg. Biomembr., 1993, 25, 137.

58 R. J. Clarke, Adv. Colloid Interface Sci., 2001, 89, 263.

59 S. Todorovics, M. M. Pereira, T. M. Bandeiras, M. Teixeira, P. Hildebrandt and D. H. Murgida, J. Am. Chem. Soc., 2005, 127, 13561. 